\title{
ВMJ Global Health Changes in respiratory diseases in South Korea during the COVID-19 pandemic: an interrupted time series study
}

\author{
Seog-Kyun Mun, ${ }^{1}$ Bo Ram Yang, ${ }^{2}$ Munyoung Chang (D) ${ }^{1}$
}

To cite: Mun S-K, Yang BR, Chang M. Changes in respiratory diseases in South Korea during the COVID-19 pandemic: an interrupted time series study. BMJ Global Health 2021;6:e006912. doi:10.1136/ bmjgh-2021-006912

Handling editor Seye Abimbola

- Additional supplemental material is published online only. To view, please visit the journal online (http://dx.doi.org/10. 1136/bmjgh-2021-006912).

Received 15 July 2021 Accepted 27 October 2021

\section{Check for updates}

(c) Author(s) (or their employer(s)) 2021. Re-use permitted under CC BY-NC. No commercial re-use. See rights and permissions. Published by BMJ.

'Department of Otorhinolaryngology-Head and Neck Surgery, Chung-Ang University College of Medicine, Seoul, South Korea

${ }^{2}$ College of Pharmacy,

Chungnam National University, Daejeon, South Korea

Correspondence to Dr Munyoung Chang; cadu01@cau.ac.kr

\section{ABSTRACT}

Introduction In South Korea, non-pharmaceutical interventions such as mask-wearing, hand washing and social distancing were strictly implemented to prevent the spread of COVID-19 after a national crisis alert was raised to the highest level early in the pandemic (23 February 2020). We aimed to investigate changes in the occurrence of respiratory diseases at the national level after the COVID-19 outbreak.

Methods The study period was from 1 January to 1 August 2019 (213 days) and from 1 January to 31 July 2020 (213 days). Based on the National Health Insurance Service data, we analysed changes in the numbers of patients treated for respiratory diseases. The changes in the numbers of individuals using public transportation and visiting the theatre were investigated to assess the effect of social distancing after the national crisis alert was raised. Differences in daily cumulative numbers (DDCNs) in 2020 were calculated as follows: (daily cumulative number in 2020)-(cumulative number for that day in 2019). A change over time in DDCNs of $<0$ was taken as indication that the numbers decreased from 2019 to 2020. Segmented regression analyses were performed using generalised least squares method to identify changes in trends of DDCNs of patients treated for respiratory diseases and individuals using public transportation and visiting the theatre.

Results After the national crisis alert was raised to the highest level, DDCNs of patients treated for respiratory diseases, individuals using public transportation, and those visiting the theatre exhibited a significant daily decline by 53.18 per 10000 inhabitants $(95 \% \mathrm{Cl}-65.86$ to -40.49$)$, 48.19 per 1000 inhabitants $(95 \% \mathrm{Cl}-62.05$ to -34.32$)$ and 25.30 per 5000 inhabitants $(95 \% \mathrm{Cl}-36.30$ to -14.30$)$, respectively, compared with before the national crisis alert was raised.

Conclusion Non-pharmaceutical interventions to prevent the spread of COVID-19 significantly reduce the incidence of respiratory diseases.

\section{BACKGROUND}

COVID-19 was first identified in December 2019 and has spread worldwide. According to the WHO, approximately 230 million

\section{WHAT IS ALREADY KNOWN?}

$\Rightarrow$ A recently published systematic review showed that it was difficult to draw solid conclusions on the effect of non-pharmaceutical interventions in preventing respiratory diseases.

\section{WHAT ARE THE NEW FINDINGS?}

$\Rightarrow$ After the COVID-19 outbreak, non-pharmaceutical interventions, including mask use, hand washing and social distancing were strictly practised in South Korea.

$\Rightarrow$ South Korea operates a National Health Insurance Service (NHIS) that covers all citizens and stores diagnostic information in a centralised database; therefore, NHIS data may be used to examine changes in disease epidemiology at a national level.

$\Rightarrow$ We investigated changes in the occurrence of respiratory diseases after the COVID-19 outbreak based on the NHIS data and found the non-pharmaceutical interventions that were implemented to prevent the spread of COVID-19 significantly reduced the incidence of respiratory diseases.

\section{WHAT DO THE NEW FINDINGS IMPLY?}

$\Rightarrow$ Considering the small number of countries worldwide with centralised national healthcare systems similar to the South Korean NHIS, our study provides valuable insight into the effects of nonpharmaceutical interventions on the incidence of respiratory diseases at the national level; these results may serve as strong evidence in support of policies requiring non-pharmaceutical interventions to prevent respiratory diseases.

individuals have been infected, with the death toll exceeding 4 million as of mid-September 2021. To prevent the spread of COVID-19, non-pharmaceutical interventions such as face mask use, hand washing and social distancing are recommended in most countries, which may prevent human-to-human infections by preventing transmission of aerosols or large droplets released by infected individuals. ${ }^{1}$ These measures have advantages in 

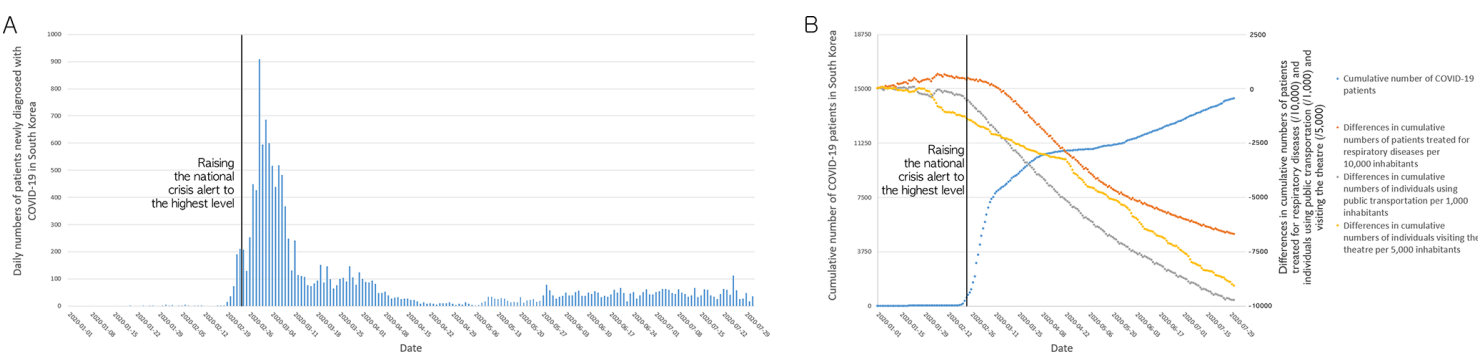

Figure 1 The daily number of patients newly diagnosed with COVID-19 and changes in numbers of patients treated for respiratory diseases and individuals using public transportation and visiting the theatre in South Korea during the COVID-19 pandemic. (A) As large-scale group infections occurred in mid-February 2020, the South Korean government raised the national crisis alert to the highest level on 23 February 2020. (B) The daily cumulative numbers of patients treated for respiratory diseases in 2020 started to decrease compared with those in 2019 after raising of the national crisis alert to the highest level. As the cumulative number of patients with COVID-19 increased, the differences in cumulative numbers of patients treated for respiratory diseases per 10000 inhabitants and individuals using public transportation per 1000 inhabitants and visiting the theatre per 5000 inhabitants decreased.

controlling upper and lower respiratory tract infections, such as rapid implementation regardless of the infection source and effectiveness against novel virus variants. Several studies have reported the effects of these nonpharmaceutical interventions for prevention of respiratory tract infections. ${ }^{1-7}$ In addition, it has been reported that wearing a mask reduces the symptoms of allergic rhinitis, one of the most common respiratory diseases, ${ }^{8-11}$ so it is known that non-pharmaceutical interventions may have an influence on the prevalence of respiratory diseases. However, a recently published systematic review reported that it was difficult to draw solid conclusions on the effectiveness of non-pharmaceutical interventions on the prevention of respiratory diseases. ${ }^{12}$ Largescale studies are needed to address the effects of nonpharmaceutical interventions.

In South Korea, the first COVID-19 case was diagnosed on 20 January $2020 .{ }^{13}$ In mid-February, large-scale group infections occurred in Daegu city (figure 1). On 23 February 2020, the South Korean government raised the national crisis alert to the highest level (level 4) and mandated non-pharmaceutical interventions such as mask use, hand washing and social distancing. Sachs $e t$ al compared the effectiveness of responses to COVID-19 in Organisation for Economic Co-operation and Development countries based on results between March and May 2020. The mortality rate, effective reproduction rate (average number of individuals infected by one infected individual) and efficiency of epidemic control were used to calculate the COVID-19 Index of Epidemic Control. South Korea ranked first with a score of 0.90 , exemplifying good implementation of non-pharmaceutical interventions to prevent COVID-19 transmission. ${ }^{14}$ Since South Korea has strict implementation of non-pharmaceutical interventions, it would appear to be the ideal country to test this effectiveness.

South Korea operates a National Health Insurance Service (NHIS) that includes all citizens and stores diagnostic information regarding medical treatment in a centralised database. ${ }^{15}$ Therefore, South Korean NHIS data may be used to examine changes in disease epidemiology at a national level. Because the implementation of non-pharmaceutical interventions may affect the prevalence of respiratory diseases other than COVID19 , we used the NHIS data to analyse changes in numbers of patients treated for respiratory diseases during the enforcement of social distancing after the national crisis alert was raised to the highest level. The changes in the numbers of individuals using public transportation and visiting the theatre were evaluated to find out whether social distancing, which is part of non-pharmaceutical interventions, was well implemented after the national crisis alert was raised.

We aimed to investigate changes in the occurrence of respiratory diseases after the COVID-19 outbreak in order to evaluate the effects of non-pharmaceutical interventions on respiratory diseases at the national level in South Korea. Given the small number of countries worldwide with centralised national healthcare databases, such as the South Korean NHIS that includes all citizens, our study provides valuable insight into the effects of national interventions on the occurrence of respiratory diseases at the population level.

\section{METHODS \\ Study design}

The study period was from 1 January to 1 August 2019 (213 days) and from 1 January to 31 July 2020 (213 days). The study comprised three parts: (1) identification of COVID-19 outbreak trends, (2) analysis of changes in cumulative incidence of patients treated for respiratory diseases and individuals using public transportation and visiting the theatre, and (3) segmented regression analyses before and after the national crisis alert was raised.

Daily numbers of patients newly diagnosed with COVID-19 were obtained from 20 January to 31 July 2020. Daily cumulative numbers of patients with COVID-19 were calculated from 20 January to the relevant day to identify COVID-19 outbreak trends. Daily numbers of patients treated for respiratory diseases per 10000 inhabitants during the study period were extracted from the NHIS data. National data 
and data from seven metropolitan cities (Seoul, Busan, Daegu, Incheon, Gwangju, Daejeon and Ulsan) and nine provinces (Gyeonggi-do, Gangwon-do, Chungcheongbuk-do, Chungcheongnam-do, Jeollabuk-do, Jeollanam-do, Gyeongsangbuk-do, Gyeongsangnam-do and Jeju-do) were collected. Implementation of social distancing was evaluated via analyses of societal movement and social activities. Daily numbers of individuals using public transportation per 1000 inhabitants and visiting the theatre per 5000 inhabitants during the study period were obtained.

\section{COVID-19 infections}

The daily numbers of patients newly diagnosed with COVID-19 in South Korea were obtained from a website operated by the Korea Disease Control and Prevention Agency. ${ }^{16}$

\section{Respiratory diseases}

NHIS provides data on the daily number of patients treated for the following respiratory diseases as the primary diagnosis, which are common in South Korea. These respiratory diseases were identified based on the Korean Classification of Disease codes as follows: acute bronchitis (J20), acute tonsillitis (J03), acute upper respiratory infections (J06), vasomotor or allergic rhinitis (J30), acute pharyngitis (J02), acute nasopharyngitis (J00), acute sinusitis (J01), acute laryngitis or tracheitis (J04), bronchitis (J40), chronic sinusitis (J32), acute bronchiolitis (J21), chronic rhinitis, nasopharyngitis or pharyngitis (J31), bronchopneumonia (J18), disorders of nose and nasal sinuses (J34), peritonsillar abscess (J36) and acute lower respiratory infections (J22). These data were obtained from the Public Data Portal website operated by the Korea Information Society Agency (https://www.data.go.kr/).

Population numbers by year and region were obtained from the Korean Statistical Information Service website operated by Statistics Korea (https://kosis.kr/index/index. do). Daily numbers of patients treated for the aforementioned respiratory diseases per 10000 inhabitants were calculated from 1 January to 31 July 2020 . Information for the same time frame in 2019 (1 January-1 August 2019, to account for the leap year) was obtained.

\section{Social distancing}

The degree of social distancing in South Korea was assessed based on societal movement and social activities. The numbers of individuals using public transportation or visiting the theatre were employed as an index of the degree of implementation of social distancing. In Seoul, the capital of South Korea, the rate of transportation card usage reached $99.02 \%$ as of $2014 .{ }^{17}$ Therefore, transportation card usage information is considered a good proxy of public transportation use. The Transportation Card Big Data Integrated Information System (https://www.stcis.go.kr/wps/ main.do\#) ${ }^{18}$ is operated by the Ministry of Land, Infrastructure and Transport and the Korea Transportation Safety Authority. Daily numbers of individuals using public transportation per 1000 inhabitants nationwide from 1 January
2020 to 31 July 2020 and from 1 January to 1 August 2019 were obtained from this website, which provides statistics on national transportation card data.

Daily numbers of individuals visiting the theatre per 5000 inhabitants from 1 January to 31 July 2020 and from 1 January to 1 August 2019 were obtained from the Korean box-office information system operated by the Korean film council (https://www.kobis.or.kr/ kobis/business/stat/them/findDailyTotalList.do), which aggregates ticket issuance information for movie theatres nationwide and provides daily numbers of individuals visiting the theatre. ${ }^{19}$

\section{Analyses}

Daily numbers of patients treated for respiratory diseases and individuals using public transportation and visiting the theatre in 2020 were divided into two groups based on the timing of the highest-level national crisis alert: group 2020-1 comprised the period from 1 January to 22 February 2020, and group 2020-2 comprised the period from 23 February to 31 July 2020. Daily 2019 numbers were divided into two groups according to similar time frames. Group 2019-1 comprised the period from 1 January to 22 February 2019, and group 2019-2 comprised the period from 23 February to 1 August 2019 (to account for the leap year). The differences in cumulative incidence over the entire period of each group between group 2019-1 and group 2020-1 and between group 2019-2 and group 2020-2 were tested using Poisson test.

Daily cumulative numbers were calculated from 1 January of each year to the corresponding day in 2019 and 2020. Differences in daily cumulative numbers (DDCNs) in 2020 were calculated as follows: (daily cumulative number in 2020)-(cumulative number for that day in 2019) (figure 2).$^{20} \mathrm{~A}$ change over time in DDCNs of $<0$ was taken as an indication that the numbers of patients treated for respiratory diseases or individuals using public transportation or visiting the theatre decreased from 2019 to 2020. In contrast, a change over time in DDCNs of $>0$ was taken as an indication of the opposite trend.

A segmented regression model was employed to evaluate changes in trends of DDCNs in 2020 following the highest-level national crisis alert according to the following equation ${ }^{21}$ :

$$
\begin{aligned}
Y_{t}=\beta_{0}+\beta_{1} \times & \text { Time_baseline }+\beta_{2} \times \text { national_alert }+\beta_{3} \\
& \times \text { Time_national_alert }+\varepsilon
\end{aligned}
$$

where $Y_{t}$ represents the DDCNs of patients treated for respiratory diseases (per 10 000) or individuals using public transportation (per 1000) or visiting the theatre (per 5000), and Time_baseline indicates the number of days as a continuous variable from commencement of the study period (1-213). national_alert is a dummy variable representing the number of days before (national_ alert $=0$ ) and after (national_alert=1) the national crisis alert was raised (1 January-22 February 2020 and 23 February-31 July 2020, respectively). Time_national_alert is a continuous variable indicating the number of days 


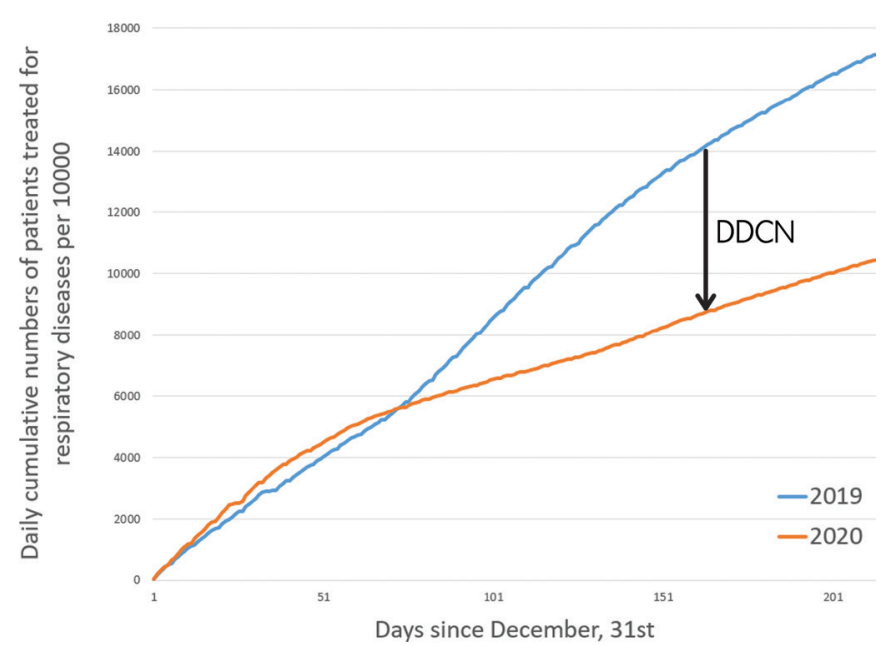

Figure 2 The concept of DDCNs. DDCNs in 2020 were calculated as follows: (daily cumulative number in 2020)(cumulative number for that day in 2019). A change over time in DDCNs of $<0$ was taken as an indication that the numbers of patients treated for respiratory diseases or individuals using public transportation or visiting the theatre decreased from 2019 to 2020. In contrast, a change over time in DDCNs of $>0$ was taken as an indication of the opposite trend.

DDCN, difference in daily cumulative numbers.

after the national crisis alert was raised and was set to 0 before this timepoint. The coefficient $\beta_{0}$ estimates the base level at the point of study commencement; $\beta_{1}$ estimates the changes in DDCNs over time (baseline trend) before the national crisis alert was raised, $\beta_{2}$ estimates the immediate changes in DDCNs (level change) after the national crisis alert was raised; $\beta_{3}$ estimates the temporal changes in trends (trend change) of DDCNs after the national crisis alert was raised; and $\varepsilon$ estimates the random error. Autocorrelation between error terms was assessed with the Durbin-Watson statistic. Values ranged from 0.014 to 0.044 and demonstrated autocorrelation. The correlation structure was estimated through autocorrelation function and partial autocorrelation function analysis. We then developed segmented regression models using generalised least squares method with the 'nlme' package of $\mathrm{R}$ software. These models were compared using the generic anova() function. The model with the lowest Akaike information criterion was chosen as the final model.

There were no missing data. All tests were two-tailed, and $p$ values of $<0.05$ were considered statistically significant. Statistical analyses were performed using R V.4.0.5 ( $\mathrm{R}$ Foundation for Statistical Computing, Vienna, Austria) and SAS V.9.4.

\section{Patient and public involvement}

Patients were not involved in this study.

\section{RESULTS}

\section{COVID-19 outbreak trends}

The number of patients newly diagnosed with COVID-19 and the cumulative number of patients with COVID-19 in South Korea are presented in figure 1. As the number of patients with COVID-19 surged in mid-February, the raising of the national crisis alert led to a decrease in the number of patients with newly diagnosed COVID-19. The number of daily occurrences then remained below 100 until the end of the study period.

\section{Cumulative incidence of patients treated for respiratory diseases and social distancing patterns}

The daily number of patients treated for respiratory diseases (per 10000 ) and individuals using public transportation (per 1000) and visiting the theatre (per 5000) are presented in table 1 and online supplemental tables 1 and 2. The cumulative incidence over the entire period of each group was calculated and compared between group 2019-1 and group 2020-1 and between group 2019-2 and group 2020-2 (table 2).

Before the national crisis alert was raised to the highest level, the cumulative incidence of patients treated for respiratory diseases in 2020 (group 2020-1) was 1.11 times the cumulative incidence of 2019 (group 2019-1) at the national level (95\% CI 1.06 to $1.16, \mathrm{p}<0.001$ ). The cumulative incidence of 2020 was significantly higher than that of 2019 in all regions except for Gwangju. The cumulative incidence of individuals using public transportation and visiting the theatre in 2020 (group 2020-1) was 0.95 times ( $95 \%$ CI 0.93 to $0.98, \mathrm{p}=0.001$ ) and 0.64 times $(95 \%$ CI 0.60 to $0.67, \mathrm{p}<0.001)$ the cumulative incidence of 2019 (group 2019-1), respectively.

After the national crisis alert was raised to the highest level, the cumulative incidence of patients treated for respiratory diseases in 2020 (group 2020-2) was 0.45 times the cumulative incidence of 2019 (group 2019-2) at the national level (95\% CI 0.43 to $0.46, \mathrm{p}<0.001)$. The cumulative incidence of 2020 was significantly lower than that of 2019 in all regions. The cumulative incidence of individuals using public transportation and visiting the theatre in 2020 (group 2020-2) was 0.71 times $(95 \%$ CI 0.70 to $0.73, \mathrm{p}<0.001)$ and 0.15 times $(95 \%$ CI 0.15 to $0.16, \mathrm{p}<0.001$ ) the cumulative incidence of 2019 (group 2019-2), respectively.

\section{Segmented regression model}

Estimates from segmented regression analyses of trends in DDCNs of patients treated for respiratory diseases per 10000 inhabitants and individuals using public transportation per 1000 inhabitants and visiting the theatre per 5000 inhabitants are summarised in table 3 and figure 3 . Before the national crisis alert was raised, DDCNs of patients treated for respiratory diseases nationwide showed no significant change over time. When analysed by region, no significant change over time was observed in all regions except for Daejeon and Chungcheongnam-do. Daejeon and Chungcheongnam-do showed significantly increasing changes over time (13.38 per 10000 inhabitants, $95 \%$ CI 2.62 to 24.13 , and 13.10 per 10000 inhabitants, $95 \%$ CI, 0.51 to 25.70 , respectively). After the national crisis alert was raised, DDCNs of patients treated 


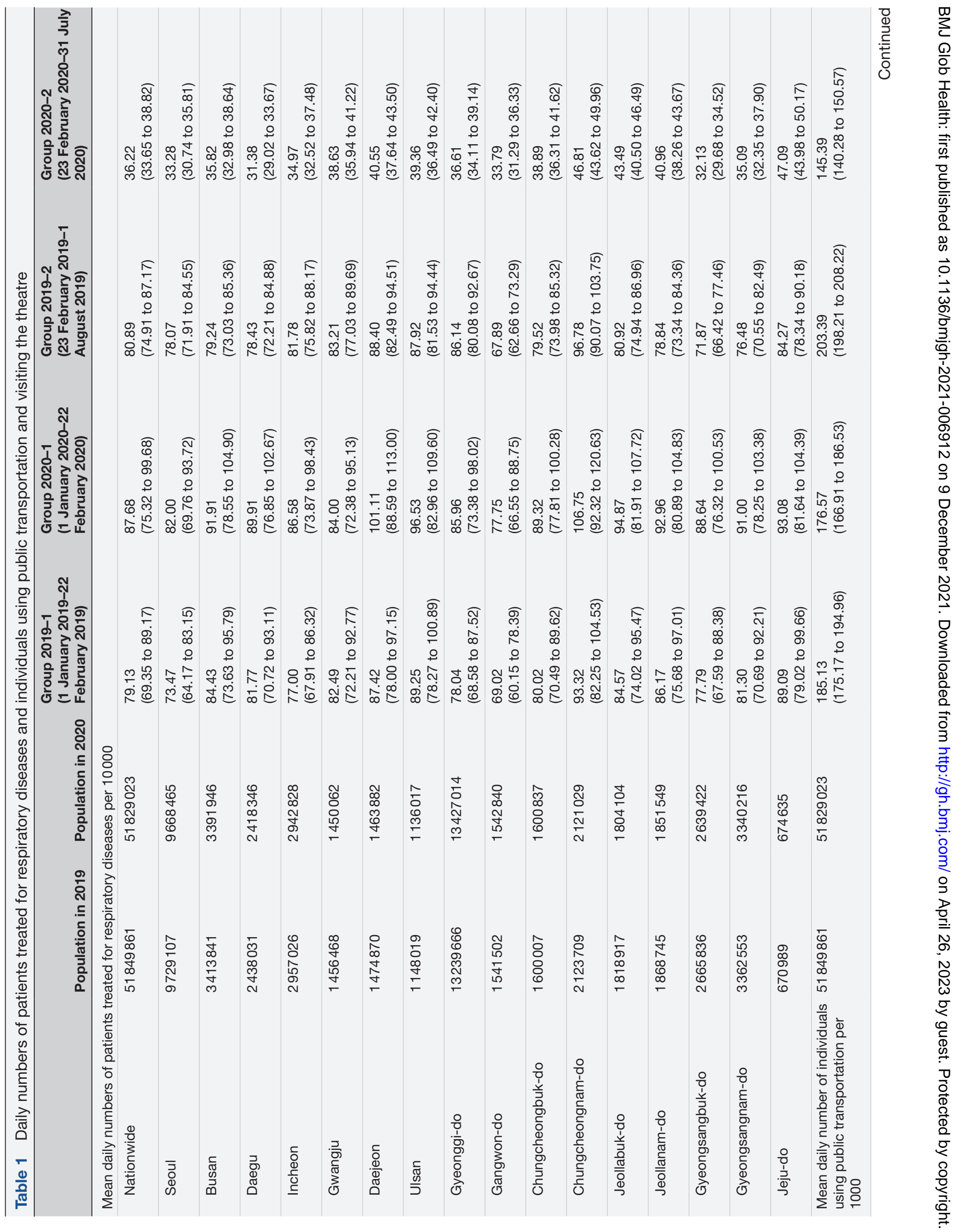




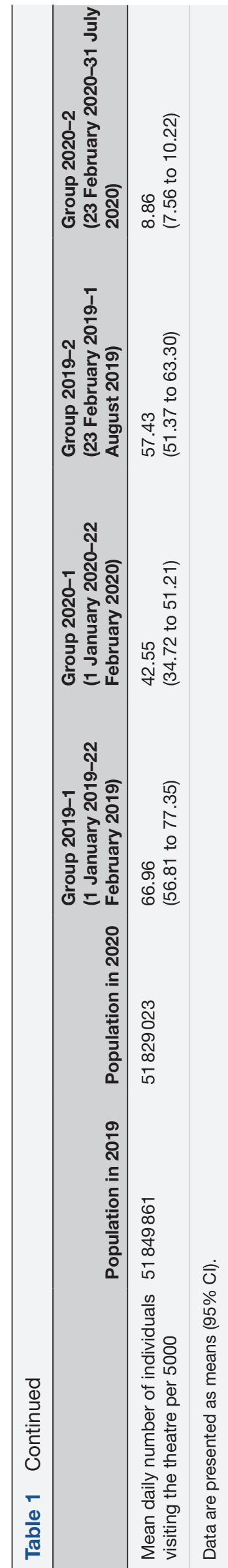

for respiratory diseases nationwide declined significantly each day, by 53.18 per 10000 inhabitants (95\% CI -65.86 to -40.49 ) compared with that before the national crisis alert was raised. Extrapolation of baseline trends resulted in an expected reduction of 8505.74 (95\% CI -9303.57 to -7707.90) in DDCNs of patients treated for respiratory diseases nationwide per 10000 inhabitants by 31 July 2020. After the national crisis alert was raised, DDCNs of patients treated for respiratory diseases declined significantly each day by $40.80-63.45$ per 10000 inhabitants in all regions compared with that before the national crisis alert was raised. Extrapolation of baseline trends resulted in an expected reduction of 6530.09-10 144.90 in DDCNs of patients treated for respiratory diseases in all regions per 10000 inhabitants by 31 July 2020 .

DDCNs of individuals using public transportation declined significantly each day by 48.19 per 1000 inhabitants $(95 \%$ CI -62.05 to -34.32$)$ compared with that before the national crisis alert was raised. Extrapolation of baseline trends resulted in an expected reduction of 7753.42 (95\% CI -8587.58 to -6919.27 ) in DDCNs of individuals using public transportation per 1000 inhabitants by 31 July 2020 .

DDCNs of individuals visiting the theatre declined significantly each day by 25.30 per 5000 inhabitants (95\% CI -36.30 to -14.30 ) compared with that before the national crisis alert was raised. Extrapolation of baseline trends resulted in an expected reduction of 4066.19 (95\% CI -4809.21 to -3323.17) in DDCNs of individuals visiting the theatre per 5000 inhabitants by 31 July 2020 .

\section{DISCUSSION}

Most countries have implemented non-pharmaceutical interventions to reduce the spread of COVID-19. The Gallup International Association surveyed 24652 adults in 28 countries worldwide between 11 and 22 March 2020 regarding the implementation of non-pharmaceutical interventions, including mask use, hand washing and social distancing. ${ }^{22}$ South Korea reported the highest rates of medical mask use (94\%), hand washing (92\%) and staying at home/less social interaction (85\%) among the surveyed countries, indicating strong nonpharmaceutical intervention practices.

Our results confirm that social distancing was more strongly practiced in South Korea after the national crisis alert was raised to the highest level. Compared with the same period in 2019, the cumulative incidence of individuals using public transportation in 2020 was significantly lower, and the use of public transportation decreased with a steeper declining trend after the national crisis alert was raised to the highest level. This suggests that social contact decreased or that person-to-person contact decreased due to the use of private cars instead of public transportation. Our findings indicate that the number of individuals visiting the theatre, a representative leisure activity in South Korea, also decreased after this timepoint. However, the number of theatre visits had begun 


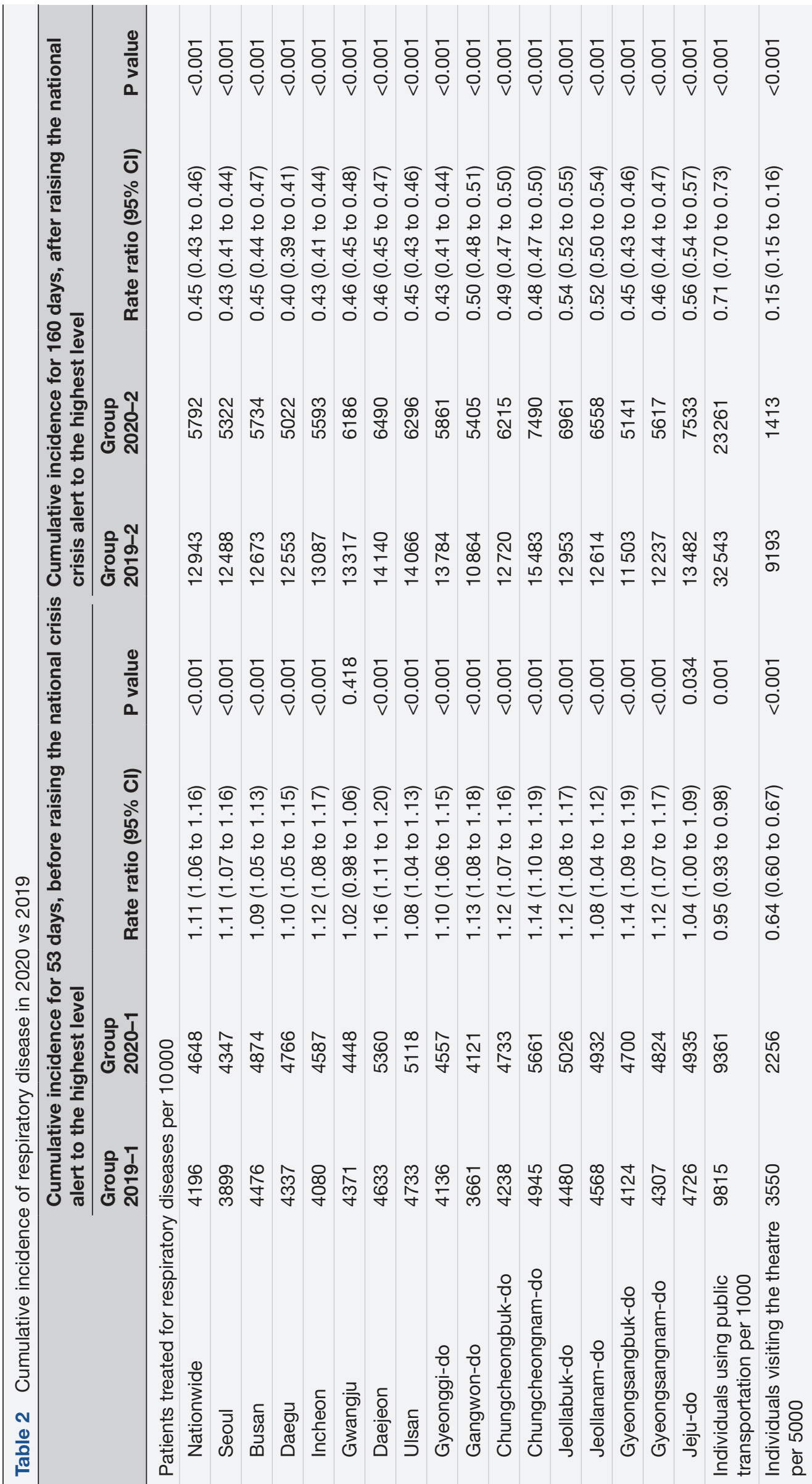




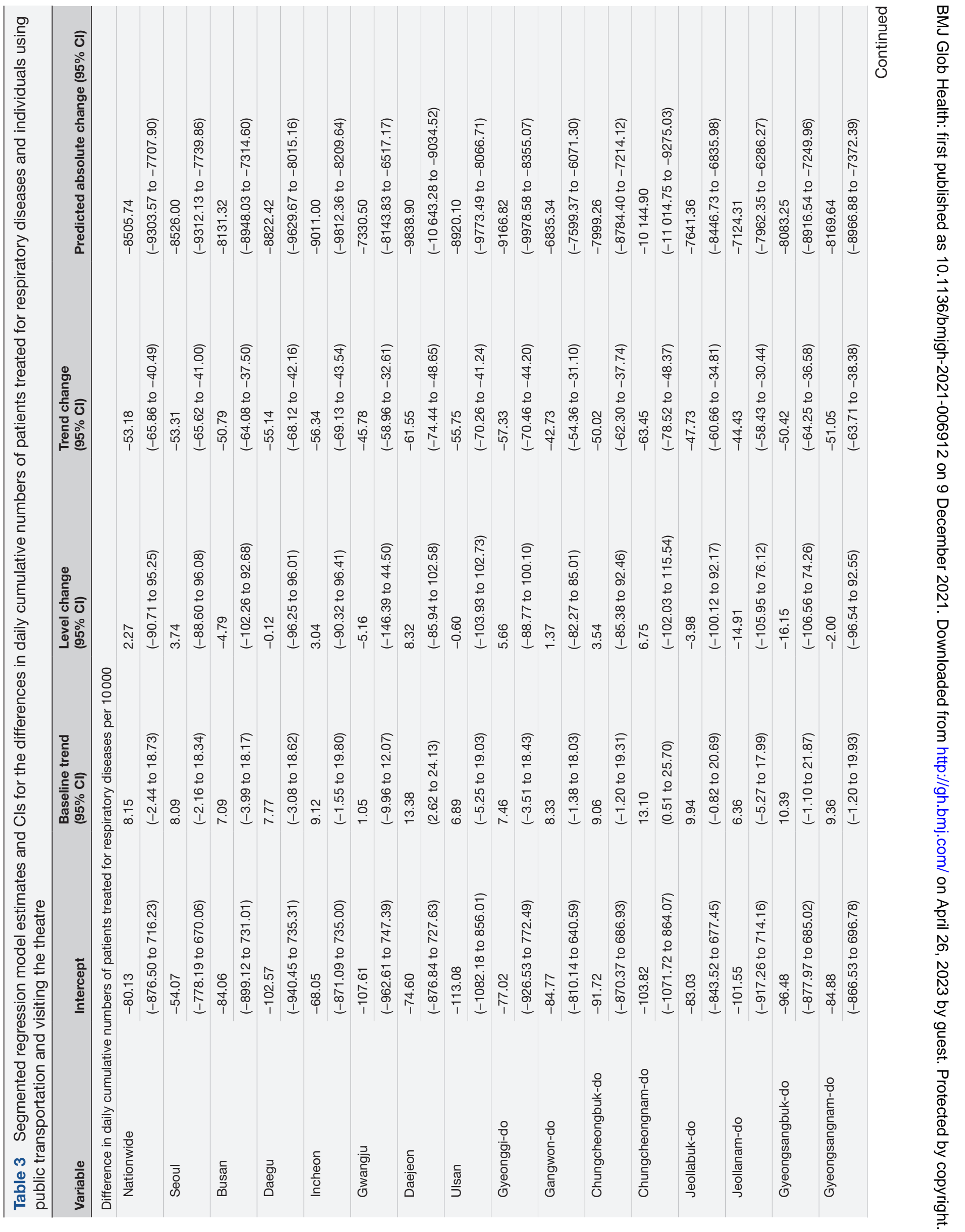




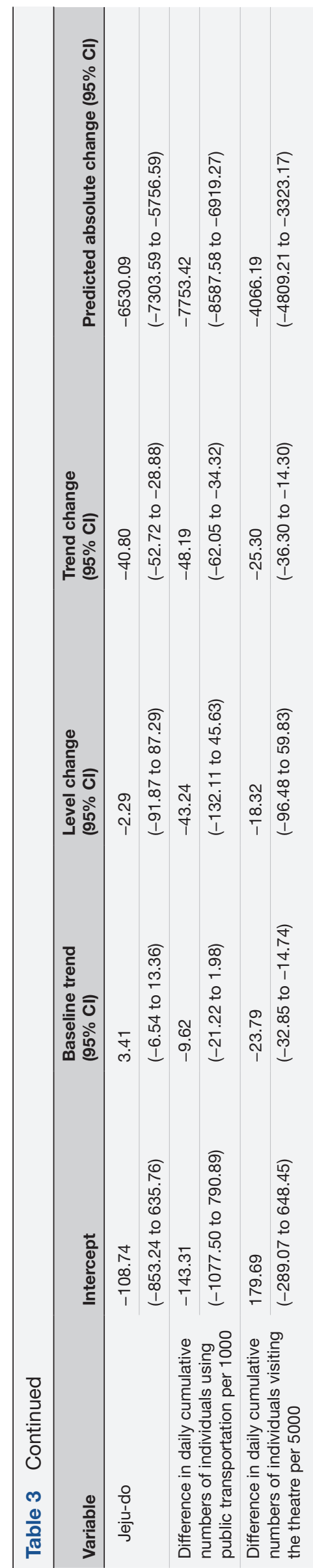

to decline in 2020 compared with that in 2019 before this timepoint. This may have been due to avoidance of theatre visits as a result of the COVID-19 pandemic even before the national crisis alert was raised, given the less essential nature of theatre visits compared with public transportation use. Nevertheless, the steeper decline in the number of theatre visits after the national crisis alert was raised indicates the stronger enforcement of social distancing after this timepoint. These results suggest that non-pharmaceutical interventions such as social distancing were well implemented in South Korea.

We used the NHIS data to examine changes in respiratory disease occurrence at the national level. Despite the COVID-19 outbreak, the cumulative incidence of patients treated for respiratory diseases in 2020 initially was significantly higher than that in 2019 , and the number of patients treated for respiratory diseases tended to increase in 2020 before the national crisis alert was raised. However, after the national crisis alert was raised, the cumulative incidence of patients treated for respiratory diseases in 2020 was significantly lower than that in 2019, and the number of patients with respiratory diseases declined in 2020 compared with 2019. Enforcement of non-pharmaceutical interventions to prevent COVID-19 transmission may have suppressed other respiratory diseases.

Several studies have investigated the inhibitory effects of non-pharmaceutical interventions on the incidence of respiratory diseases. Regarding respiratory tract infections, Aiello et al divided 1437 young adults into three groups (face mask use, face masks with hand hygiene or control) during the influenza season and observed that the incidence of influenza-like illness was significantly lower in the group that used face masks with hand hygiene. ${ }^{1}$ Cowling et al divided influenza-infected individuals into three groups (control, hand hygiene or surgical face masks plus hand hygiene) and reported that transmission between family members was lower in the group that used face masks plus hand hygiene. ${ }^{3}$ Carabin $e t$ al and Ladegaards and Stage reported that the incidence of respiratory tract infections was reduced by repeated hand washing. ${ }^{25}$ However, the effectiveness of non-pharmaceutical interventions for inhibiting respiratory tract infections remains controversial. Jacobs et al reported that the use of face masks did not affect the incidence of cold symptoms in hospital workers, ${ }^{23}$ although their sample size was small $(n=32)$. A recently published systematic review reported that there was uncertainty about the effects of face masks, but hand hygiene was likely to modestly reduce the burden of respiratory tract infections. ${ }^{12}$ Zhang et al reported that mask-wearing was effective for preventing person-to-person COVID-19 transmission in Italy and New York City and argued that social distancing is also important. ${ }^{7}$ Lee et al reported that non-pharmaceutical interventions implemented after the COVID-19 outbreak reduced seasonal influenza incidence in South Korea. ${ }^{24}$ Collectively, these results support the use of non-pharmaceutical interventions such as hand 
A

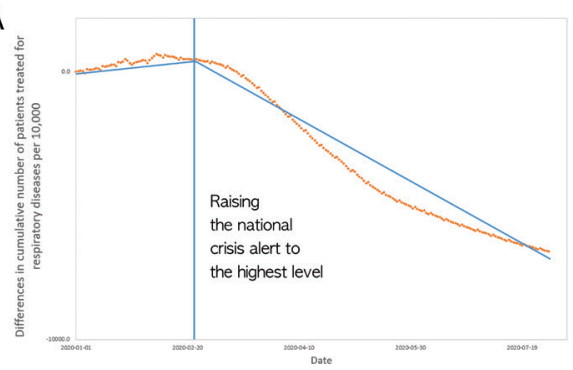

B

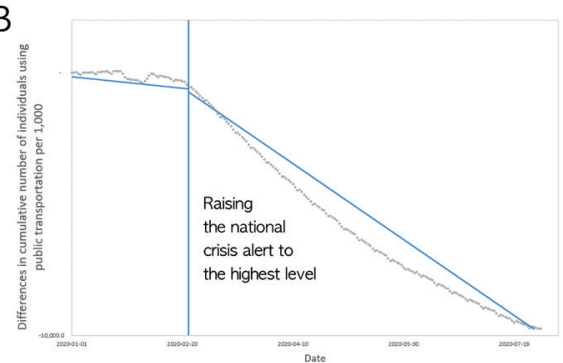

c

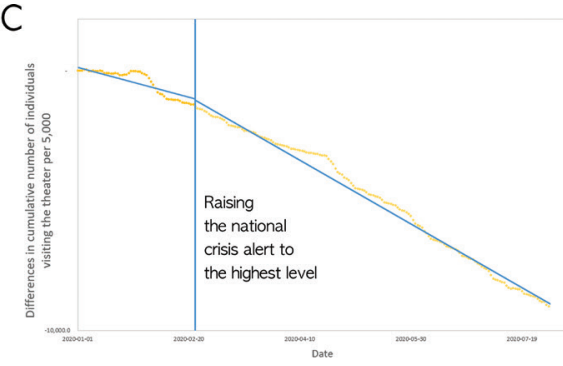

Figure 3 Changes in trends in numbers of patients treated for respiratory diseases and individuals using public transportation and visiting the theatre. After the national crisis alert was raised to the highest level, the differences in cumulative numbers of (A) patients treated for respiratory diseases per 10000 inhabitants and individuals using (B) public transportation per 1000 inhabitants and (C) visiting the theatre per 5000 inhabitants declined, compared with the previous year.

hygiene, wearing masks and social distancing to suppress respiratory tract infections. Regarding allergic rhinitis, Dror et al reported that the symptoms of allergic rhinitis decreased after wearing a mask during the COVID-19 pandemic. ${ }^{8}$ This was presumed to be because wearing a mask reduced allergen inhalation and exposure. ${ }^{25}$ This is consistent with our results.

Our study has some limitations. We estimated a decrease in respiratory disease incidence based on the decrease in the number of patients treated for respiratory diseases. However, as respiratory diseases included in our study are relatively mild diseases that are common in South Korea, the number of patients treated for these respiratory diseases may have decreased as patients were reluctant to visit hospitals due to fear of getting the COVID-19 outbreak. A recent study on changes in severe respiratory diseases during the non-pharmaceutical intervention period in South Korea used the NHIS data in the same way as in our study. ${ }^{4}$ This study reported that the cumulative incidence of hospital admissions for chronic obstructive pulmonary disease (COPD) and asthma during the non-pharmaceutical intervention period for COVID-19 decreased to $58 \%$ and $48 \%$ of the previous period, respectively. Since severe respiratory diseases require treatment in hospital, it is unlikely that patients will not visit the hospital for fear of getting COVID-19. Respiratory diseases included in our study decreased to $45 \%$ of that in the previous period, not vastly different from hospital admissions for COPD and asthma. Therefore, it is judged that avoidance of the hospital visits for fear of COVID-19 infection was not the cause of the decrease in the number of patients treated for respiratory diseases.

\section{CONCLUSIONS}

Our study demonstrates that the incidence of respiratory diseases decreased after the national crisis alert was raised to the highest level in South Korea. Our results suggest that non-pharmaceutical interventions implemented to prevent COVID-19 spread reduced the incidence of other respiratory diseases. This study provides valuable insight into the effectiveness of implementing non-pharmaceutical interventions at the national level, as manifested by a reduced nationwide incidence of respiratory diseases. These results may serve as strong evidence in support of policies requiring nonpharmaceutical interventions to prevent respiratory diseases.

Contributors $\mathrm{MC}$ accepts full responsibility for the finished work and the conduct of the study, had access to the data, and controlled the decision to publish as as the guarantor. MC and S-KM conceptualised and designed the study, led the data collection and analysis, and wrote the manuscript. MC, BRY and S-KM contributed to study design. BRY advised on the data analyses and provided overall oversight of the study. All authors read and approved the final manuscript.

Funding The authors have not declared a specific grant for this research from any funding agency in the public, commercial or not-for-profit sectors.

Competing interests None declared.

Patient consent for publication Not applicable.

Ethics approval Ethics approval was obtained from the institutional review board of Chung-Ang University Hospital (2101-004-19348). The requirement for written informed consent was waived, given that anonymised data were used. All research was performed in accordance with the Declaration of Helsinki.

Provenance and peer review Not commissioned; externally peer reviewed.

Data availability statement Data are available upon reasonable request. This study used NHIS data made by National Health Insurance Service (NHIS). The data used in this study are available from the Public Data Portal website operated by the Korea Information Society Agency (https://www.data.go.kr/), Korean Statistical Information Service website operated by Statistics Korea (https://kosis.kr/index/ index.do), Transportation Card Big Data Integrated Information System operated by the Ministry of Land, Infrastructure and Transport and the Korea Transportation Safety Authority (https://www.stcis.go.kr/wps/main.do\#), and Korean box-office information system operated by the Korean film council (https://www.kobis.or.kr/ kobis/business/stat/them/findDailyTotalList.do).

Supplemental material This content has been supplied by the author(s). It has not been vetted by BMJ Publishing Group Limited (BMJ) and may not have been peer-reviewed. Any opinions or recommendations discussed are solely those of the author(s) and are not endorsed by BMJ. BMJ disclaims all liability and responsibility arising from any reliance placed on the content. Where the content includes any translated material, BMJ does not warrant the accuracy and reliability of the translations (including but not limited to local regulations, clinical guidelines, terminology, drug names and drug dosages), and is not responsible for any error and/or omissions arising from translation and adaptation or otherwise.

Open access This is an open access article distributed in accordance with the Creative Commons Attribution Non Commercial (CC BY-NC 4.0) license, which permits others to distribute, remix, adapt, build upon this work noncommercially, and license their derivative works on different terms, provided the original work is properly cited, appropriate credit is given, any changes made indicated, and the use is non-commercial. See: http://creativecommons.org/ licenses/by-nc/4.0/.

ORCID iD

Munyoung Chang http://orcid.org/0000-0003-0136-3893 


\section{REFERENCES}

1 Aiello AE, Murray GF, Perez V, et al. Mask use, hand hygiene, and seasonal influenza-like illness among young adults: a randomized intervention trial. J Infect Dis 2010;201:491-8.

2 Carabin H, Gyorkos TW, Soto JC, et al. Effectiveness of a training program in reducing infections in toddlers attending day care centers. Epidemiology 1999;10:219-27.

3 Cowling BJ, Chan K-H, Fang VJ, et al. Facemasks and hand hygiene to prevent influenza transmission in households: a cluster randomized trial. Ann Intern Med 2009;151:437-46.

4 Huh K, Kim Y-E, Ji W, et al. Decrease in hospital admissions for respiratory diseases during the COVID-19 pandemic: a nationwide claims study. Thorax 2021;76:939-41.

5 Ladegaard MB, Stage V. [Hand-hygiene and sickness among small children attending day care centers. An intervention study]. Ugeskr Laeger 1999;161:4396-400.

6 Larson EL, Ferng Y-hui, Wong-McLoughlin J, et al. Impact of nonpharmaceutical interventions on URIs and influenza in crowded, urban households. Public Health Rep 2010;125:178-91.

7 Zhang R, Li Y, Zhang AL, et al. Identifying airborne transmission as the dominant route for the spread of COVID-19. Proc Natl Acad Sci U S A 2020;117:14857-63.

8 Dror AA, Eisenbach N, Marshak T, et al. Reduction of allergic rhinitis symptoms with face mask usage during the COVID-19 pandemic. J Allergy Clin Immunol Pract 2020;8:3590-3.

9 Oberg T, Brosseau LM. Surgical mask filter and fit performance. Am $J$ Infect Control 2008;36:276-82.

10 Portnoy J, Barnes C. Clinical relevance of spore and pollen counts. Immunol Allergy Clin North Am 2003;23:vi:389-410.

11 Seidman MD, Gurgel RK, Lin SY, et al. Clinical practice guideline: allergic rhinitis. Otolaryngol Head Neck Surg 2015;152:S1-43.

12 Jefferson T, Del Mar CB, Dooley L, et al. Physical interventions to interrupt or reduce the spread of respiratory viruses. Cochrane Database Syst Rev 2020;11:Cd006207.

13 Korean Society of Infectious Diseases, Korean Society of Pediatric Infectious Diseases, Korean Society of Epidemiology, et al. Report on the epidemiological features of coronavirus disease 2019 (COVID-19) outbreak in the Republic of Korea from January 19 to March 2, 2020. J Korean Med Sci 2020;35:e112.

14 Sachs J, Schmidt-Traub G, Kroll C. Sustainable development report 2020: the sustainable development goals and COVID-19. Cambridge: Cambridge University Press, 2020.
15 Kim JA, Yoon S, Kim LY, et al. Towards Actualizing the value potential of Korea health insurance review and assessment (HIRA) data as a resource for health research: strengths, limitations, applications, and strategies for optimal use of HIRA data. J Korean Med Sci 2017;32:718-28.

16 Korea Disease Control and Prevention Agency. Press release: coronavirus infectious Disease-19 outbreak in Korea. Cheongju-si, South Korea: Korea Disease Control and Prevention Agency, 2020. https://www.cdc.go.kr/index.es?sid=a3

17 Korea Research Institute for Human Settlements. Land policy: traffic analysis in the metropolitan area using public transportation card data. Sejong-si, South Korea: Korea Research Institute for Human Settlements, 2020. https://www.krihs.re.kr/issue/cbriefView2.do? seq $=23391$

18 Ministry of Land, Infrastructure and Transport and the Korea Transportation Safety Authority. Numbers of individuals using public transportation. Kimcheon-si, South Korea: Ministry of Land, Infrastructure and Transport and the Korea Transportation Safety Authority, 2020. https://www.stcis.go.kr/wps/main.do\#

19 Korean box-office information system under the Korean film council. Total audience and sales. Busan, South Korea: Korean box-office information system under the Korean film council, 2020. https:// www.kobis.or.kr/kobis/business/stat/them/findDailyTotalList.do

20 Baldi E, Sechi GM, Mare C, et al. COVID-19 kills at home: the close relationship between the epidemic and the increase of out-ofhospital cardiac arrests. Eur Heart J 2020;41:3045-54.

21 Wagner AK, Soumerai SB, Zhang F, et al. Segmented regression analysis of interrupted time series studies in medication use research. J Clin Pharm Ther 2002;27:299-309.

22 Gallup International Association. The coronavirus: a vast scared majority around the world. Zurich, Switzerland: Gallup international association, 2020. Available: https://www.gallup.co. kr.gallupdb,fileDownload

23 Jacobs JL, Ohde S, Takahashi O, et al. Use of surgical face masks to reduce the incidence of the common cold among health care workers in Japan: a randomized controlled trial. Am J Infect Control 2009;37:417-9.

24 Lee $\mathrm{H}$, Lee $\mathrm{H}$, Song $\mathrm{K}-\mathrm{H}$, et al. Impact of public health interventions on seasonal influenza activity during the COVID-19 outbreak in Korea. Clin Infect Dis 2021;73:e132-e140.

25 Cherrie JW, Apsley A, Cowie H, et al. Effectiveness of face masks used to protect Beijing residents against particulate air pollution. Occup Environ Med 2018;75:446-52. 